\title{
IDENTIFICAÇÃO DE COMPOSTOS VOLÁTEIS DA CÚRCUMA EMPREGANDO MICROEXTRAÇÃO POR FASE SÓLIDA E CROMATOGRAFIA GASOSA ACOPLADA À ESPECTROMETRIA DE MASSAS ${ }^{1}$
}

\author{
Adriana, R. MATA², David L. NELSON², Robson, J. C. F. AFONSO ${ }^{3}$,
}

\author{
Maria Beatriz A. GLÓRIA², Roberto, G. JUNQUEIRA,*
}

\begin{abstract}
RESUMO
Compostos voláteis da cúrcuma (Curcuma longa L.) cultivada no Brasil foram isolados por microextração por fase sólida. Os rizomas foram cozidos em solução de bicarbonato de sódio $0,1 \%$, fatiados, secos e triturados. Visando estabelecer o sistema ideal para a microextração, fibras de polidimetilsiloxano de $100 \mu \mathrm{m}$ de espessura foram expostas ao headspace de frascos de $10 \mathrm{~mL}$. Estudou-se a influência das seguintes variáveis sobre o rendimento dos compostos voláteis obtidos: amostras em pó $(0,1$ a $1,0 \mathrm{~g})$ e em solução $(40 \mathrm{mg} / \mathrm{L})$, diferentes temperaturas $\left(40\right.$ a $\left.70^{\circ} \mathrm{C}\right)$ e tempos $(2$ a $20 \mathrm{~min})$ de partição. O efeito da temperatura $\left(210\right.$ a $\left.240^{\circ} \mathrm{C}\right)$ e do tempo (3 e $\left.5 \mathrm{~min}\right)$ de dessorção também foi avaliado. As melhores condições para a partição dos compostos voláteis foram $0,1 \mathrm{~g}$ do pó, $70^{\circ} \mathrm{C}$ e $5 \mathrm{~min}$. A temperatura de $220^{\circ} \mathrm{C}$ e o tempo de 5 minutos foram os de maior eficiência para a dessorção. A cromatografia gasosa foi conduzida em coluna capilar, detecção por ionização de chama e identificação por espectrometria de massas. A análise dos espectros de massas obtidos para os nove compostos voláteis predominantes indicou a presença de ar-curcúmeno, ar-turmerona, zingibereno, $\beta$-sesquifelandreno, sabineno, 1,8-cineol e 1,4-terpineol.
\end{abstract}

Palavras-chave: Curcuma longa; compostos voláteis; microextração por fase sólida; cromatografia gasosa; espectrometria de massa.

\section{SUMMARY}

IDENTIFICATION OF VOLATILE COMPOUNDS OF TURMERIC USING SOLID PHASE MICROEXTRACTION AND GAS CHROMATOGRAPHY COUPLED TO MASS SPECTROMETRY. Volatile compounds from turmeric (Curcuma longa L.) cultived in Brazil were isolated by solid phase microextraction. The rhizomes were cooked in $0.1 \%$ sodium bicarbonate solution, sliced, dried and ground. Polydimethyldiloxanes fibers (100 mm diameter) were exposed to the headspace of $10 \mathrm{~mL}$ flasks with the aim of establishing an ideal microextraction system. The influence of the following parameters on the volatile substances obtained was studied: powder samples (0.1 to $1.0 \mathrm{~g})$ and dissolved samples $(40 \mathrm{mg} / \mathrm{L})$, different temperatures $\left(40\right.$ to $\left.70^{\circ} \mathrm{C}\right)$ and partition periods $(2$ to $20 \mathrm{~min})$. The effect of temperature $\left(210\right.$ to $\left.240^{\circ} \mathrm{C}\right)$ and time (3 to $5 \mathrm{~min}$ ) on desorption efficiency was also evaluated. Optimum conditions for the partition of the volatiles were $0.1 \mathrm{~g}$ of powder sample, $70^{\circ} \mathrm{C}$ and $5 \mathrm{~min}$. A temperature of $220^{\circ} \mathrm{C}$ and a $5 \mathrm{~min}$ period were of better efficiency for desorption. Gas chromatography was performed using capillary column and flame ionization detector. The eluates were identified by mass spectrometry. The mass spectra obtained for the predominant volatile compounds indicated the presence of ar-curcumene, ar-tumerone, zingeberene, $\beta$ sesquiphellandrene, sabinene, 1,8-cineol and 1,4-terpineol.

Keywords: Curcuma longa; volatile compounds; SPME; GC; GC-MS.

\section{1 - INTRODUÇÃO}

A Curcuma longa, pertencente à família Zingiberaceae, subordem Zingiberoidae, é originária da Índia e está distribuída pela China, Oriente Médio, Formosa, Indonésia, Java, Filipinas, Caribe, Norte da Austrália e América do Sul [7]. No Brasil, a cúrcuma é confundida com o açafrão verdadeiro (Crucus sativus), planta de clima mediterrâneo, não cultivada no país. A cúrcuma e o açafrão são empregados na culinária, com objetivos semelhantes. A cúrcuma foi introduzida no país por volta de 1980, resultando em boa produtividade, devido às condições climáticas ideais para o seu cultivo [14]. O emprego da cúrcuma em alimentos como sopas desidratadas, molhos, produtos cárneos e de panificação, sobremesas à base de ovos e queijo prato, dentre outros, advém das substâncias flavorizantes presentes e responsáveis por seu odor característico, além de seu poder corante [9, 10, 14].

\footnotetext{
1. Recebido para publicação em 02/07/2003. Aceito para publicação em 01/11/2003 (001158).

2. Departamento de Alimentos, Faculdade de Farmácia, UFMG. E-mail: junkeira@dedalus,lcc.ufmg.br

${ }^{3}$ Fundação Centro Tecnológico de Minas Gerais

*A quem a correspondência deve ser enviada.
}

KELKAR \& RAO [8] relataram que os componentes do óleo volátil dos rizomas da cúrcuma, resultantes de uma destilação por arraste de vapor, são predominantemente cetonas e álcoois sesquiterpênicos. Dentre estes, identificaram $\alpha$-D-felandreno, D-sabineno, cineol, borneol, zingibereno e turmeronas. Segundo OGUNTIMEIN et al. [13], os principais constituintes do óleo volátil do rizoma são os sesquiterpenos cetônicos turmerona e ar-turmerona. ZWAVING \& BOS [24] verificaram a presença de limoneno, 1,8-cineol, linalol, borneol em espécies de cúrcuma. RICHARD \& LOO [16] relataram turmerona, ar-turmerona, $\alpha$ - e $\beta$-zingibereno, 1,8cineol, $\alpha$-felandreno, sabineno e borneol, como os principais constituintes do óleo volátil do rizoma da cúrcuma.

Pesquisas sobre a composição do óleo volátil da cúrcuma, principalmente a cultivada na Índia, são descritas na literatura. Entretanto, poucos trabalhos foram desenvolvidos até o momento com relação aos compostos voláteis da cúrcuma brasileira. Interesse mais expressivo vem sendo dado aos pigmentos curcuminóides, de ampla utilização no processamento de diversos alimentos [4, 10, 21].

Muitos métodos têm sido empregados para análise de flavorizantes presentes nos alimentos. Entretanto, a maioria consome muito tempo de análise, requerendo concentração da amostra, bem como o emprego de 
solventes [1, 6, 24]. Mais recentemente, a extração com fluidos supercríticos, associada à cromatografia, também vem sendo aplicada na análise de pigmentos e óleos voláteis da cúrcuma. $\mathrm{O}$ uso de $\mathrm{CO}_{2}$ como solvente resultou na extração de turmeronas e outros compostos voláteis de baixa polaridade [11, 12].

Outra técnica empregada na extração de compostos voláteis de alimentos, desenvolvida em 1990 por ARTHUR \& PAWLISZYN [3], é a denominada microextração por fase sólida (SPME - solid phase microextraction). A unidade de SPME consiste de uma fibra de sílica recoberta com material polimérico típico usado como fase estacionária e colunas cromatográficas tais como: carbowax, poliacrilato e polidimetilsiloxano. A extração fundamenta-se no equilíbrio de partição (polidimetilsiloxano) ou adsorção (poliacrilato) entre a fibra e os componentes da amostra ou seu headspace. Quando o equilíbrio é alcançado, a quantidade de composto extraído está diretamente relacionada à afinidade com a fase da fibra e sua concentração na amostra. Os compostos são dessorvidos termicamente, separados, identificados e quantificados posteriormente. Esta técnica apresenta muitas vantagens, como simplicidade, rapidez, não utilizar solventes, ter baixo custo e pode ser aplicada à análise de flavor de amostras sólidas, líquidas e gasosas, especialmente na triagem de compostos voláteis [2, 23]. Separação, identificação e quantificação são geralmente feitas por cromatografia gasosa (CG) com detector de ionização de chama e a confirmação por espectrometria de massas [6, 19].

Este trabalho teve como objetivos otimizar a extração dos compostos voláteis da Curcuma longa cultivada no Brasil por microextração em fase sólida, a separação destes por cromatografia gasosa empregando detector de ionização de chama e a identificação dos mesmos por cromatografia gasosa acoplada à espectrometria de massas.

\section{2 - MATERIAL E MÉTODOS}

\section{1 - Amostras}

\subsection{1 - Processamento}

Os rizomas de cúrcuma provenientes de Patos de Minas, Estado de Minas Gerais, Brasil, foram selecionados e limpos. Em seguida, foi realizado o cozimento dos rizomas com a casca, por 40 minutos em solução de bicarbonato de sódio 0,1\%, pH 8,6. Seguiu-se o fatiamento longitudinal dos rizomas, a secagem em estufa com circulação de ar a $40 \pm 5^{\circ} \mathrm{C}$, a trituração em microprocessador (Arno) e a tamisação (tamis malha 42). O pó obtido foi acondicionado em embalagem de vidro e armazenado $\mathrm{a}-18^{\circ} \mathrm{C}$ até o momento da análise.

\subsection{2 - Preparo das soluções das amostras}

As soluções das amostras foram preparadas empregando os solventes etanol e água segundo SANTOS, GALCERAN \& FRAISSE [18]. A concentração da solu-

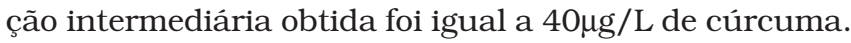
Esta solução foi utilizada nos estudos de otimização da extração e separação dos compostos voláteis da cúrcuma.

\section{2 - Padrões}

Padrões de borneol, limoneno e linalol, adquiridos da Merck (Darmstadt, Alemanha), foram utilizados para o preparo das soluções padrões. A partir de uma solução estoque de cada padrão em etanol p.a. (1g/L), prepararam-se as soluções de trabalho em água Milli-Q filtrada em membrana de $0,45 \mu \mathrm{m}$, cujas concentrações variaram de 30 a $1000 \mu \mathrm{g} / \mathrm{L}$. Soluções de trabalho do pool destes padrões, nas mesmas condições, também foram preparadas, seguindo os mesmos procedimentos. As soluções de trabalho dos padrões foram empregadas nos estudos de otimização da extração e separação dos compostos voláteis da cúrcuma.

\section{3 - Procedimentos analíticos}

\subsection{1 - Estudo das condições ideais para a se- paração dos compostos voláteis da cúrcuma}

Na extração, empregou-se o método de SPME com fibra de polidimetilsiloxano de $100 \mu \mathrm{m}$ para partição dos compostos voláteis presentes na amostra e nas soluções dos padrões. A fibra foi exposta ao headspace do frasco aquecido a $40^{\circ} \mathrm{C}$, contendo $0,1 \mathrm{~g}$ do pó, no caso das amostras e $1,0 \mathrm{~mL}$ da solução de trabalho do pool de padrões. A partição foi realizada em agitador magnético e sob aquecimento à temperatura constante de $40^{\circ} \mathrm{C}$ no interior do frasco, utilizando um equipamento Fisatom. Após 5 minutos de exposição à fibra, a seringa foi imediatamente levada ao injetor do cromatógrafo, no qual os compostos voláteis foram dessorvidos a $220^{\circ} \mathrm{C}$, por 5 minutos.

A separação dos compostos voláteis da cúrcuma foi realizada com base nas condições descritas por ZWAVING \& BOS [24] e SHARMA et al. [19], empregando um sistema para CG Shimadzu (CG-17A) equipado com detector de ionização de chama. As condições cromatográficas empregadas foram coluna capilar DB-1 $(25 \mathrm{~m}$

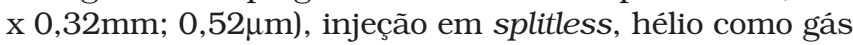
de arraste e temperatura do injetor de $220^{\circ} \mathrm{C}$ e do detector de $250^{\circ} \mathrm{C}$. As condições ideais para a programação de temperatura da coluna foram investigadas.

\subsection{2- Estudo das condições de partição e dessorção}

O estudo da otimização da partição e dessorção dos voláteis baseou-se nos procedimentos adotados por YANG \& PEPPARD [23] e SANTOS, GALCERAN \& FRAISSE [18]. Testes preliminares foram realizados a fim de verificar o tempo e a temperatura a serem empregados na partição e dessorção dos compostos voláteis da cúrcuma. Para a partição, o tempo foi de 5 minutos e as temperaturas de 40,60 e $70^{\circ} \mathrm{C}$ foram avaliadas. Na dessorção, foram estudados os tempos de 3 e 5 minutos e as temperaturas de 210,220 e $240^{\circ} \mathrm{C}$. 


\subsection{3 - Estudo da linearidade das respostas das soluções padrões}

A linearidade das respostas das soluções padrões na extração, empregando a técnica SPME, foi verificada e baseou-se no método descrito por SANTOS GALCERAN \& FRAISSE [18]. Para este estudo, foram utilizadas as soluções de trabalho do pool dos padrões. O erro experimental inerente a esta etapa, devido a possiveis variações no processo de extração, foi avaliado pelo ensaio em triplicata, sendo a variabilidade para cada concentração de padrão calculada em termos de coeficiente de variação. Neste estudo, a partição foi realizada com $1,0 \mathrm{~mL}$ da solução do pool de padrões, com agitação magnética a $70 \pm 2{ }^{\circ} \mathrm{C}$ por 5 minutos e a dessorção ocorreu a $220^{\circ} \mathrm{C}$ por 5 minutos. As condições cromatográficas empregadas foram as mencionadas anteriormente.

\subsection{4 - Estudo do tempo ideal para partição}

O tempo ideal para a partição dos compostos voláteis da cúrcuma foi estabelecido com base nas curvas de saturação/equilíbrio para os padrões de borneol, limoneno e linalol. Utilizou-se $1,0 \mathrm{~mL}$ da solução de trabalho do pool dos padrões (600 $\mu \mathrm{g} / \mathrm{L}$ de cada padrão). As condições de partição, dessorção e cromatografia foram as mesmas mencionadas no item anterior, excetuando-se a exposição da fibra ao headspace do frasco, realizada nos tempos de 2; 5; 7; 10; 15 e 20 minutos. A análise foi realizada em cinco repetições para verificar a variabilidade referente a esta etapa.

\subsection{5 - Estudo das condições ótimas de partição}

Visando estabelecer as condições ideais de partição, testes em cinco repetições foram realizados, empregando amostras para diferentes temperaturas. Neste estudo, utilizaram-se amostras em pó $(0,1 ; 0,5$ e 1,0g) e em solução $(1,0 \mathrm{~mL})$ contendo $40 \mu \mathrm{g} / \mathrm{L}$ de cúrcuma. A influência da condição da amostra (pó ou solução) e das temperaturas 40,60 e $70^{\circ} \mathrm{C}$ na extração dos nove compostos predominantes foi avaliada. As demais condições de partição, bem como as de dessorção e cromatográficas usadas foram as mencionadas anteriormente.

\subsection{6 - Identificação dos compostos voláteis}

A identificação dos compostos voláteis da cúrcuma foi baseada nos espectros obtidos na espectrometria de massas com ionização por elétrons a $70 \mathrm{eV}$ na faixa de 40 a 650 daltons, realizada em equipamento GCQ Finnigan CG/EM com analisador do tipo ion trap. Os espectros de massas foram comparados com dados da biblioteca de espectros NIST do equipamento e na dedução dos fragmentos obtidos. As condições experimentais empregadas foram as seguintes: coluna capi-

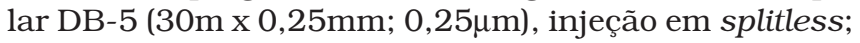
gás hélio de arraste; programação de temperatura da coluna de $40^{\circ} \mathrm{C}$ por $5 \mathrm{~min}$, aumento de $8^{\circ} \mathrm{C} / \mathrm{min}$ até $130^{\circ} \mathrm{C}$, aumento de $30^{\circ} \mathrm{C} / \mathrm{min}$ até $200^{\circ} \mathrm{C}$ e manutenção a $200^{\circ} \mathrm{C}$ por $5 \mathrm{~min}$; temperatura do injetor de $220^{\circ} \mathrm{C}$ e do detector de $250^{\circ} \mathrm{C}$.

\section{4 - Análise estatística}

$\mathrm{Na}$ otimização da partição foi adotado o delineamento fatorial $3 \times 4$ em cinco repetições, avaliando-se a influência de três temperaturas e quatro condições de extração dos voláteis. As médias dos tratamentos foram comparadas pelo teste de Duncan, a 5\% de probabilidade [15].

\section{3 - RESULTADOS E DISCUSSÃO}

\section{1 - Otimização da separação dos compostos vo- láteis da cúrcuma}

$\mathrm{Na}$ otimização da metodologia para separação dos compostos voláteis da cúrcuma foram consideradas as resoluções dos nove picos predominantes na amostra. Também foi considerado o comportamento dos picos dos padrões de borneol, limoneno e linalol, compostos voláteis que foram encontrados por ZWAVING \& BOS [24] no óleo volátil da cúrcuma cultivada na Índia.

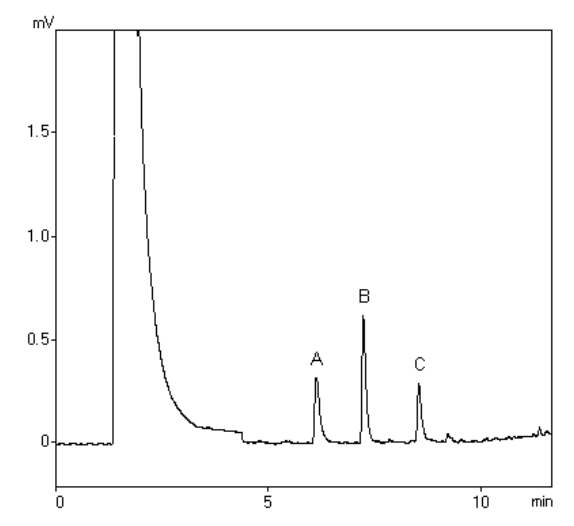

(a)

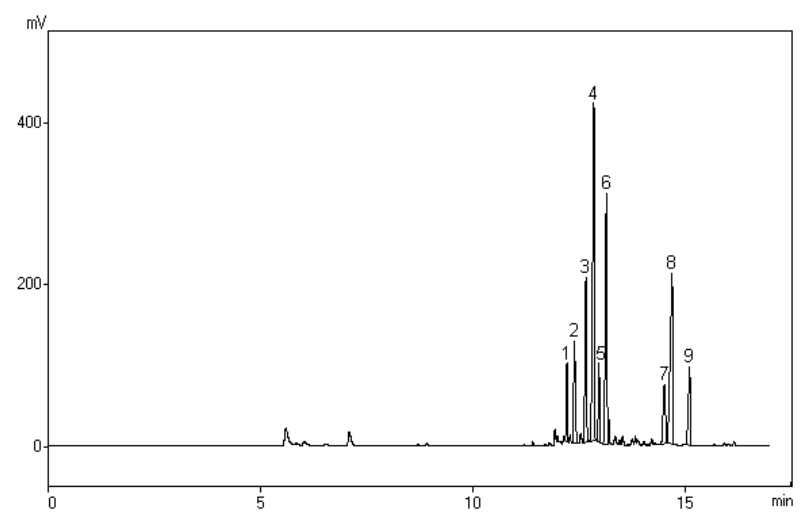

(b)

Condições cromatográficas: coluna DB-1 (25m x 0,32mm; 0,52mm); gás de arraste hélio; detector de ionização de chama; temperatura do injetor $220^{\circ} \mathrm{C}$, do detector $250^{\circ} \mathrm{C}$ e da coluna $80^{\circ} \mathrm{C}$ por $2 \mathrm{~min}$, aumento de $8^{\circ} \mathrm{C} / \mathrm{min}$ até $130^{\circ} \mathrm{C}, 130^{\circ} \mathrm{C}$ por lmin, nantes da cúrcuma foram numerados de 1 a 9 , em ordem crescente do tempo de retenção

FIGURA 1. Perfil cromatográfico (a) dos padrões de limoneno (A), linalol (B) e borneol (C) e (b) dos compostos voláteis da cúrcuma.

De acordo com observações dos cromatogramas obtidos para cada teste, verificou-se que a condição 
para programação de temperatura da coluna que melhor separou os picos dos padrões e os nove picos predominantes das amostras foi manter a $80^{\circ} \mathrm{C}$ por $2 \mathrm{~min}$, taxa de aquecimento de $8^{\circ} \mathrm{C} / \mathrm{min}$ até $130^{\circ} \mathrm{C}$, deixar a $130^{\circ} \mathrm{C}$ por $1 \mathrm{~min}$, aumentar $30^{\circ} \mathrm{C} / \mathrm{min}$ até $200^{\circ} \mathrm{C}$ e manter a $200^{\circ} \mathrm{C}$ por $5 \mathrm{~min}$. Cromatogramas típicos nesta condição de separação dos padrões de borneol, limoneno e linalol e dos voláteis da cúrcuma estão apresentados na Figura 1.

\section{2 - Otimização das condições de partição e dessorção}

\subsection{1 - Emprego dos padrões de borneol, limoneno e linalo1}

Conforme os estudos realizados para a escolha das condições ideais de dessorção constatou-se que as temperaturas de 220 e $240^{\circ} \mathrm{C}$ não influenciaram a separação e a área dos picos analisados. No entanto, diminuição nas áreas dos picos dos padrões foi notada na temperatura de $210^{\circ} \mathrm{C}$, optando-se, desta forma, pela temperatura de $220^{\circ} \mathrm{C}$. Empregando o tempo de $3 \mathrm{~min}$, verificou-se a presença de picos remanescentes em uma segunda injeção, imediatamente após cada análise. Supôs-se que este tempo não era suficiente para a completa dessorção dos compostos voláteis da fibra. Por outro lado, no tempo de 5 minutos, não foram observados picos na segunda injeção, sendo este o tempo estabelecido. Estas condições de tempo e temperatura de dessorção foram semelhantes àquelas utilizadas por VARIYAR, GHOLAP \& THOMAS [22] na análise dos compostos voláteis do gengibre, vegetal pertencente a mesma família da cúrcuma.

Foi observada uma resposta linear da área dos picos em função da concentração dos padrões adsorvidos no processo de extração por SPME, nas faixas de concentração estudadas. Valores de coeficiente de determinação $\left(\mathrm{R}^{2}\right)$ variando de 0,9770 a 0,9917 foram obtidos. Os coeficientes de variação $(\mathrm{CV})$ observados para as diferentes concentrações dos padrões foram de 3 a $17 \%$, podendo ser considerados aceitáveis. Também SANTOS, GALCERAN \& FRAISSE [18] encontraram valores de $\mathrm{R}^{2}$ na faixa de 0,9982 a 0,9996 e CV variando de 7 a 13\% em seus estudos de otimização da técnica de SPME para análise de compostos orgânicos voláteis da água.

Observando a curva de saturação dos padrões ( $F i^{-}$ gura 2), o tempo ideal de partição estabelecido foi de $5 \mathrm{~min}$, correspondente ao rendimento máximo. Neste tempo, a área média dos picos foi duas vezes maior que a obtida no tempo inicial de $2 \mathrm{~min}$, mas acima de $7 \mathrm{~min}$ o rendimento caiu acentuadamente. ARTHUR \& PAWLISZYN [3] relataram que, em altas concentrações dos compostos, a absorção pela fibra pode decrescer com o tempo. Segundo YANG \& PEPPARD [23], a cinética de partição depende da natureza do composto de interesse, sendo influenciada pelo equilíbrio dinâmico entre três fases: a estacionária da fibra, a gasosa no inte- rior do frasco e a líquida ou sólida da amostra. Não foram encontrados dados na literatura sobre a extração de compostos voláteis da cúrcuma por SPME. Para a partição dos compostos voláteis do suco de maçã, SONG et al. [20] estabeleceram um tempo de 4 minutos. Os coeficientes de variação para cada tempo de partição de cada padrão variaram de 8 a $27 \%$. YANG \& PEPPARD [23] obtiveram valores de CV de 16,5\% para limoneno e $3,2 \%$ para linalol para amostras de sucos de frutas.

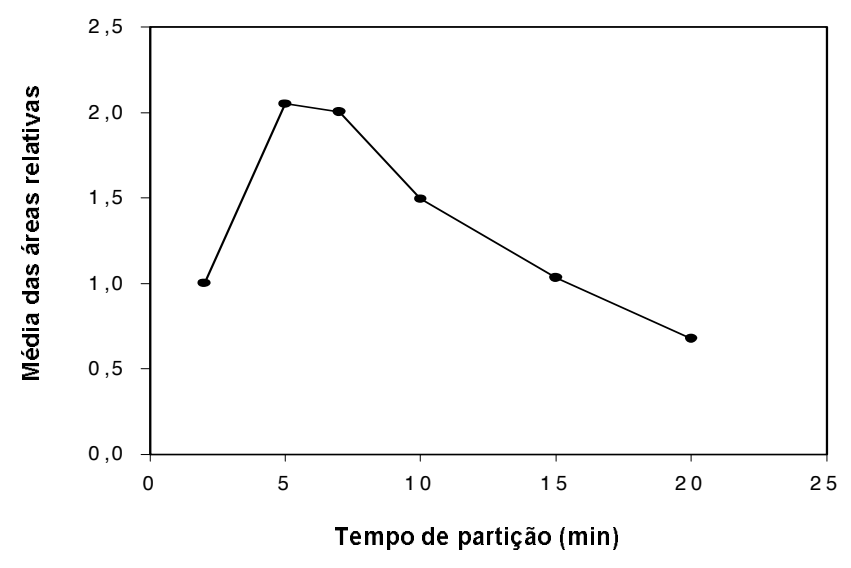

A resposta foi expressa em termos de área relativa, considerando como unitária a área no tempo igual a $2 \mathrm{~min}$ para cada padrão $(600 \mathrm{mg} / \mathrm{L})$. Condições cromatográficas: coluna DB-1 ( $25 \mathrm{~m}$ × $0,32 \mathrm{~mm}$; 0,52mm); gás de arraste hélio; detector de ionização de chama; temperatura do injetor $220^{\circ} \mathrm{C}$, do detector $250^{\circ}$ e da coluna $80^{\circ} \mathrm{C}$ por 2 min, aumento de $8^{\circ} \mathrm{C} / \mathrm{min}$ até $130^{\circ} \mathrm{C}, 130^{\circ} \mathrm{C}$ por $1 \mathrm{~min}$, aumento de
$30^{\circ} \mathrm{C} / \mathrm{min}$ até $200^{\circ} \mathrm{C}, 200^{\circ} \mathrm{C}$ por $5 \mathrm{~min}$.

FIGURA 2. Curva de saturação para a mistura dos padrões de limoneno, linalol e borneol em função do tempo de partição.

\subsubsection{Emprego de amostras de cúrcuma}

A partir das áreas de nove picos presentes, a influência da amostra em solução e em quantidades diferentes de pó na eficiência da partição foi estudada, com o objetivo de obter o maior rendimento na extração dos voláteis. YANG \& PEPPARD [23] também avaliaram a influência de diferentes quantidades da amostra $(0,5$ a $5,5 \mathrm{~mL}$ ) nas condições de partição de compostos voláteis de sucos de frutas, verificando que o mais eficiente foi $0,5 \mathrm{~mL}$.

De maneira geral, observa-se na Tabela 1 que a condição de $1,0 \mathrm{~mL}$ da solução da amostra foi a que apresentou menor eficiência e diferiu significativamente $(p<0,05)$ das amostras em pó, nas diferentes temperaturas. A temperatura de $40^{\circ} \mathrm{C}$ foi a que resultou nas menores áreas dos picos, para todas as condições da amostra. Nas temperaturas de 60 e $70^{\circ} \mathrm{C}$, houve pequena variação das áreas com relação ao tipo da amostra. No entanto, para os nove compostos estudados, a condição de extração empregando $0,1 \mathrm{~g}$ e $70^{\circ} \mathrm{C}$ foi a que apresentou maior área, diferindo significativamente das demais $(\mathrm{p}<0,05)$. 
TABELA 1. Rendimento de extração para nove compostos voláteis da cúrcuma extraídos sob diferentes condições de partição e separados por cromatografia gasosa

\begin{tabular}{|c|c|c|c|c|c|}
\hline \multirow{3}{*}{ Pico } & \multirow{3}{*}{$\begin{array}{c}\text { Temperatura } \\
\left({ }^{\circ} \mathrm{C}\right)\end{array}$} & \multicolumn{4}{|c|}{ Área dos picos nas condições de amostragem $\left(\times 10^{3}\right)^{*}$} \\
\hline & & & pó & & solução \\
\hline & & $0,1 \mathrm{~g}$ & $0,5 \mathrm{~g}$ & $1,0 \mathrm{~g}$ & $1,0 \mathrm{~mL}$ \\
\hline \multirow{3}{*}{1} & 40 & $1,4017^{\text {aby }}$ & $2,4138^{\text {ay }}$ & $2,2821^{a y}$ & $0,1427^{\mathrm{bw}}$ \\
\hline & 60 & $8,5726^{\mathrm{ax}}$ & $4,0791^{\mathrm{bx}}$ & $3,0888^{b x}$ & $0,0000^{\mathrm{cw}}$ \\
\hline & 70 & $11,2093^{\text {aw }}$ & $6,5572^{\mathrm{bw}}$ & $4,6708^{\mathrm{cw}}$ & $0,0000^{\mathrm{dv}}$ \\
\hline \multirow{3}{*}{2} & 40 & $0,0000^{b y}$ & $3,7836^{\text {ay }}$ & $3,6988^{\mathrm{ax}}$ & $0,1503^{\mathrm{bN}}$ \\
\hline & 60 & $14,6208^{\mathrm{ax}}$ & $6,7567^{\mathrm{bx}}$ & $5,2388^{b x}$ & $0,0000^{\mathrm{cw}}$ \\
\hline & 70 & $19,4266^{\text {aw }}$ & $11,4650^{\mathrm{bN}}$ & $8,3793^{\mathrm{cw}}$ & $0,0000^{\mathrm{dw}}$ \\
\hline \multirow{3}{*}{3} & 40 & $3,6175^{a y}$ & $7,6636^{\text {aw }}$ & $7,7690^{\mathrm{aw}}$ & $0,3818^{\mathrm{bN}}$ \\
\hline & 60 & $30,1279^{\mathrm{ax}}$ & $14,0243^{\mathrm{bx}}$ & $11,0649^{\mathrm{bx}}$ & $1,0100^{\mathrm{cw}}$ \\
\hline & 70 & $42,4350^{\mathrm{aw}}$ & $25,2553^{\text {by }}$ & $19,0071^{\mathrm{cx}}$ & $0,9147^{\mathrm{dN}}$ \\
\hline \multirow{3}{*}{4} & 40 & $6,4244^{a y}$ & $10,9574^{a y}$ & $10,6534^{\text {ay }}$ & $0,4582^{\mathrm{bw}}$ \\
\hline & 60 & $39,3649^{a x}$ & $18,2460^{b x}$ & $14,1762^{\mathrm{bx}}$ & $1,2004^{\mathrm{cw}}$ \\
\hline & 70 & $45,0053^{\mathrm{aw}}$ & $26,4451^{\mathrm{bN}}$ & $20,0930^{\mathrm{cw}}$ & $3,9660^{\mathrm{dv}}$ \\
\hline \multirow{3}{*}{5} & 40 & $9,1018^{\mathrm{ax}}$ & $2,2897^{\text {by }}$ & $2,3139^{\mathrm{bx}}$ & $0,1273^{\mathrm{cw}}$ \\
\hline & 60 & $8,8184^{\mathrm{ax}}$ & $4,0840^{b x}$ & $3,2269^{b x}$ & $0,0000^{\mathrm{cw}}$ \\
\hline & 70 & $11,9991^{\text {aw }}$ & $7,0876^{\mathrm{bw}}$ & $5,4022^{\mathrm{bw}}$ & $0,0000^{\mathrm{cw}}$ \\
\hline \multirow{3}{*}{6} & 40 & $7,5636^{\text {ay }}$ & $9,3071^{\text {ay }}$ & $9,5633^{\mathrm{ax}}$ & $0,5442^{\mathrm{bv}}$ \\
\hline & 60 & $34,8610^{\mathrm{ax}}$ & $16,4964^{\mathrm{bx}}$ & $13,2001^{b x}$ & $1,2307^{\mathrm{cw}}$ \\
\hline & 70 & $46,6413^{\mathrm{aw}}$ & $27,9548^{\mathrm{bw}}$ & $21,7091^{\mathrm{bN}}$ & $1,1103^{\mathrm{cw}}$ \\
\hline \multirow{3}{*}{7} & 40 & $2,8679^{a y}$ & $4,0640^{a y}$ & $5,2576^{\mathrm{ax}}$ & $0,1898^{\mathrm{bN}}$ \\
\hline & 60 & $11,3259^{\mathrm{ax}}$ & $7,6405^{b x}$ & $6,9062^{b x}$ & $0,7134^{\mathrm{cw}}$ \\
\hline & 70 & $21,1177^{\mathrm{aw}}$ & $12,7550^{\mathrm{bN}}$ & $12,9120^{\mathrm{bN}}$ & $0,7873^{\mathrm{cw}}$ \\
\hline \multirow{3}{*}{8} & 40 & $5,6894^{a y}$ & $7,1059^{a y}$ & $8,7527^{\mathrm{ax}}$ & $0,5205^{\mathrm{bv}}$ \\
\hline & 60 & $19,3566^{\mathrm{ax}}$ & $12,5087^{\mathrm{bx}}$ & $11,5339^{\mathrm{bx}}$ & $1,7141^{\mathrm{cw}}$ \\
\hline & 70 & $31,6117^{\text {aw }}$ & $18,8947^{\mathrm{b} w}$ & $18,4702^{\mathrm{bw}}$ & $1,8247^{\mathrm{cw}}$ \\
\hline \multirow{3}{*}{9} & 40 & $2,3276^{\mathrm{by}}$ & $2,9827^{\text {aby }}$ & $4,4416^{\mathrm{ax}}$ & $0,2945^{\mathrm{cw}}$ \\
\hline & 60 & $8,5767^{\mathrm{ax}}$ & $5,8723^{\mathrm{bx}}$ & $5,2856^{b x}$ & $1,0062^{\mathrm{cw}}$ \\
\hline & 70 & $15,5322^{\mathrm{aw}}$ & $10,4487^{\mathrm{bw}}$ & $9,5668^{\mathrm{bw}}$ & $1,1093^{\mathrm{cw}}$ \\
\hline
\end{tabular}

*Médias (5 repetições) indicadas pela mesma letra em uma mesma linha (a, b, c, d) ou para cada pico em uma mesma coluna $(w, x, y, z)$ não diferiram entre si pelo teste ou para cada pico em uma mesma col
de Duncan, a $5 \%$ de probabilidade.

\section{3 - Identificação de compostos voláteis da cúrcuma}

Os espectros obtidos na CG/EM, a comparação com os dados da biblioteca do instrumento e a dedução dos fragmentos indicaram a presença dos seguintes compostos voláteis nos rizomas da cúrcuma cultivada no Brasil: ar-turmerona (2-heten-4-ona, 2-metil-6-(4-metilfenil), PM 218) e 1,8-cineol (2-oxabiciclo[2,2,2] octano, 1,3,3-trimetil, PM 154) (Figura 3); ar-curcumeno (benzeno, 1-(1,5-dimetil4-hexenil)-4-metil, PM 202) e sabineno (biciclo[3,1,0] hexano,4-metileno-1-(1-metiletil), PM 136) (Figura 4); $\beta$ sesquifelandreno (ciclohexeno,3-(1,5-dimetil-4-hexenil) 6-metileno, PM 204) e 1,4-terpineol (3-ciclo-hexeno-1metano, $\alpha, \alpha, 4$-trimetil, PM 154) (Figura 5) e zingibereno (1,3-ciclohexadieno, 5-(1,5-dimetil-4-hexenil)-2-metil, PM
204) (Figura 6). Estes compostos foram observados por CHOUDHURY et al. [6], RICHMOND \& POMBO-VILLAR [17] e SHARMA et al. [19] em rizomas de cúrcuma. Os principais compostos voláteis identificados por CHATTERJEE et al. [5] na cúrcuma irradiada foram $\alpha$ felandreno, $p$-cimeno, 1,8 cineol, $\beta$-cariofileno, arcurcumeno, zingebereno, $\beta$-sesquifelandreno, nerolidol, ar-turmerona, curlona e dehidrozingerona. Segundo GOVINDARAJAN [7], os compostos ar-curcumeno e arturmerona podem ser formados durante tratamentos térmicos, aos quais a cúrcuma é submetida, podendo o arcurcúmeno também ser formado pela conversão do zingibereno e do $\beta$-sesquifelandreno durante o armazenamento. A presença de borneol, limoneno e linalol não foi confirmada na cúrcuma cultivada no Brasil pela CG/EM utilizada, embora CHOUDHURY et al. [6] tenham encontrado estes compostos na cúrcuma originária da Índia.
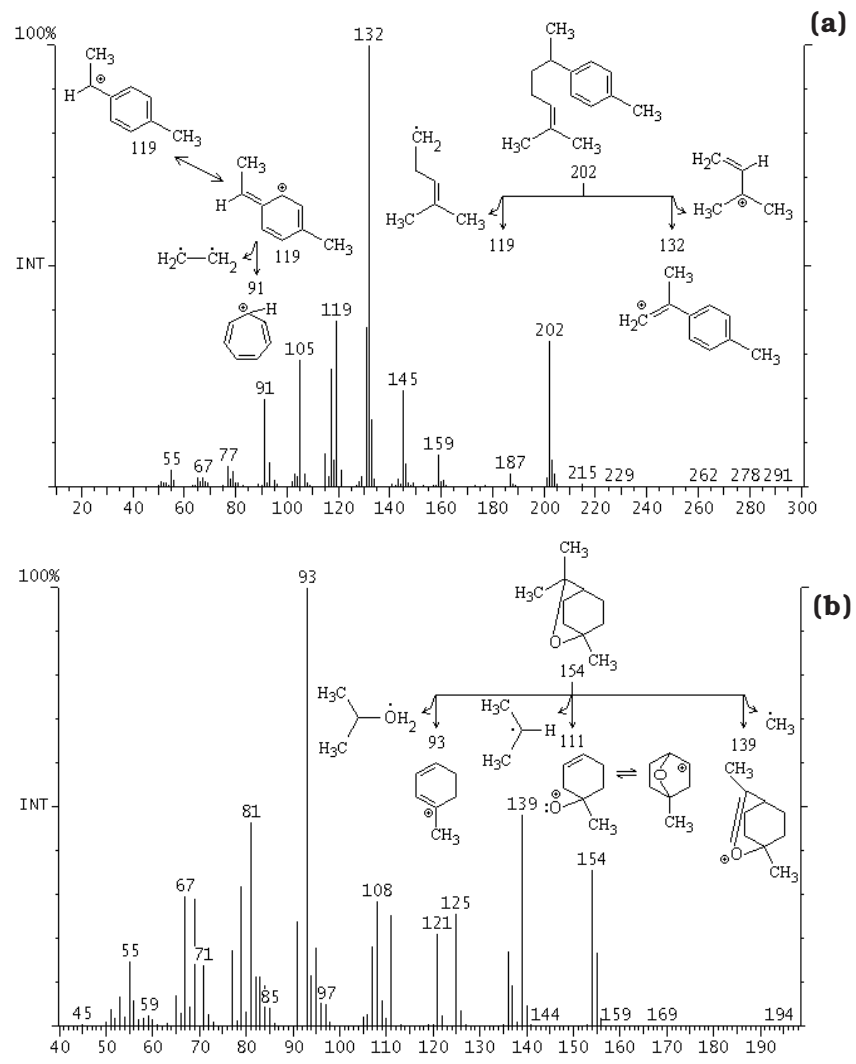

Condições cromatográficas: coluna DB-5 (30m x 0,25mm; 0,25mm); gás de arraste hélio; temperatura do injetor $220^{\circ} \mathrm{C}$, do detector $250^{\circ} \mathrm{C}$ e da coluna $40^{\circ} \mathrm{C}$ por $5 \mathrm{~min}$, aumento de $8^{\circ} \mathrm{C} / \mathrm{min}$ até $130^{\circ} \mathrm{C}$, aumento de $30^{\circ} \mathrm{C} / \mathrm{min}$ até $200^{\circ} \mathrm{C}, 200^{\circ} \mathrm{C}$ por $5 \mathrm{~min}$. $170^{\circ} \mathrm{C}$, temperatura da interface $275^{\circ} \mathrm{C}$, ionização $70 \mathrm{eV}$ e corrente de emissão $250 \mathrm{~mA}$.

FIGURA 3. Espectro de massa e possíveis fragmentos (a) da ar-turmerona e (b) do 1,8-cineol obtidos na espectrometria de massa.

A partir dos espectros de massa e cromatogramas obtidos na separação e identificação dos nove compostos voláteis predominantes nas amostras analisadas, deduziu-se que ar-curcumeno, zingibereno, $\beta$-sesquifelandreno e ar-turmerona são, respectivamente, os pi$\cos 3,4,5$ e 6 (Figura $1 b$ ). 

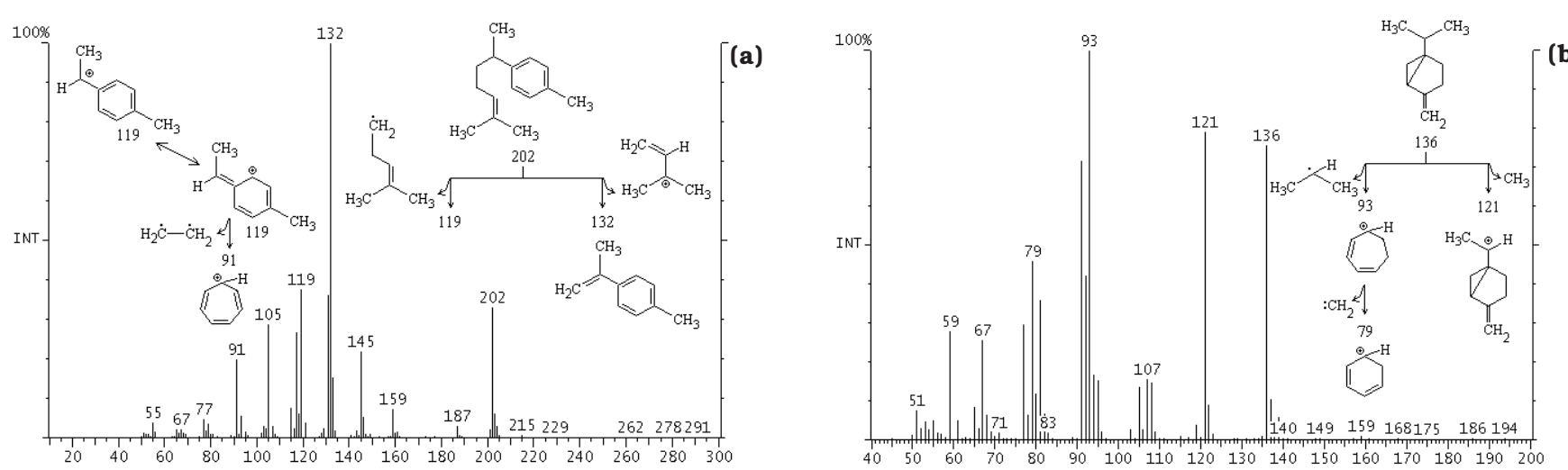

Condições cromatográficas: coluna DB- $5\left(30 \mathrm{~m}\right.$ x 0,25mm; 0,25mm); gás de arraste hélio; temperatura do injetor $220^{\circ} \mathrm{C}$, do detector $250^{\circ} \mathrm{C}$ e da coluna $40^{\circ} \mathrm{C}$ por $5 \mathrm{~min}$, aumento de $8^{\circ} \mathrm{C} / \mathrm{min}$ até $130^{\circ} \mathrm{C}$, aumento de $30^{\circ} \mathrm{C} / \mathrm{min}$ até $200^{\circ} \mathrm{C}, 200^{\circ} \mathrm{C}$ por $5 \mathrm{~min}$. Condiçốes de espectrometria de massa: detector de ion trap, temperatura da fonte $170^{\circ} \mathrm{C}$, temperatura da interface $275^{\circ} \mathrm{C}$, ionização $70 \mathrm{eV}$ e corrente de emissão $250 \mathrm{~mA}$.

FIGURA 4. Espectro de massa e possíveis fragmentos (a) do ar-curcueno e (b) do sabineno obtidos na espectrometria de massa.
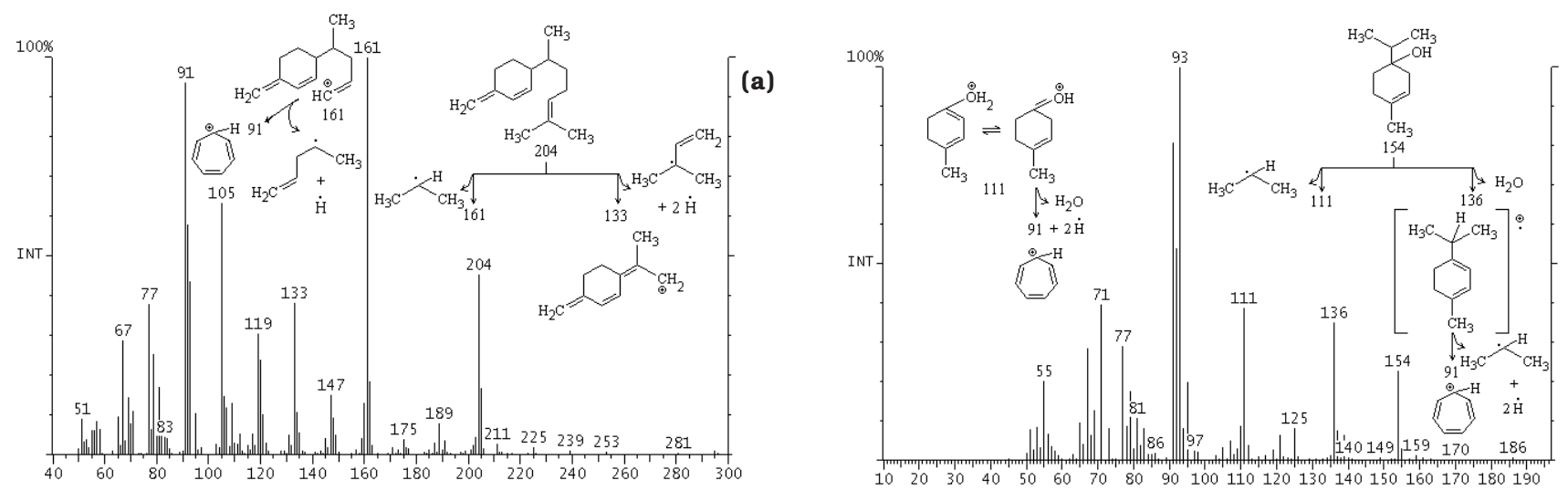
Condições cromatográficas: coluna DB- $5(30 \mathrm{~m} \times 0,25 \mathrm{~mm} ; 0,25 \mathrm{~mm})$; gás de arraste hélio; temperatura do injetor $220^{\circ} \mathrm{C}$, do detector $250^{\circ} \mathrm{C}$ e da coluna $40^{\circ} \mathrm{C}$ por 5 min, aumento
de $8^{\circ} \mathrm{C} / \mathrm{min}$ até $130^{\circ} \mathrm{C}$, aumento de $30^{\circ} \mathrm{C} / \mathrm{min}$ até $200^{\circ} \mathrm{C}, 200^{\circ} \mathrm{C}$ por 5 min. Condiçoses de espectrometria de massa: detector de ion trap, temperatura da fonte $170^{\circ} \mathrm{C}$, temperatura da interface $275^{\circ} \mathrm{C}$, ionização $70 \mathrm{eV}$ e corrente de emissão $250 \mathrm{~mA}$.

FIGURA 5. Espectro de massa e possiveis fragmentos (a) do b-sesquifelandreno e (b) do 1,4-terpineol obtidos na espectrometria de massa.

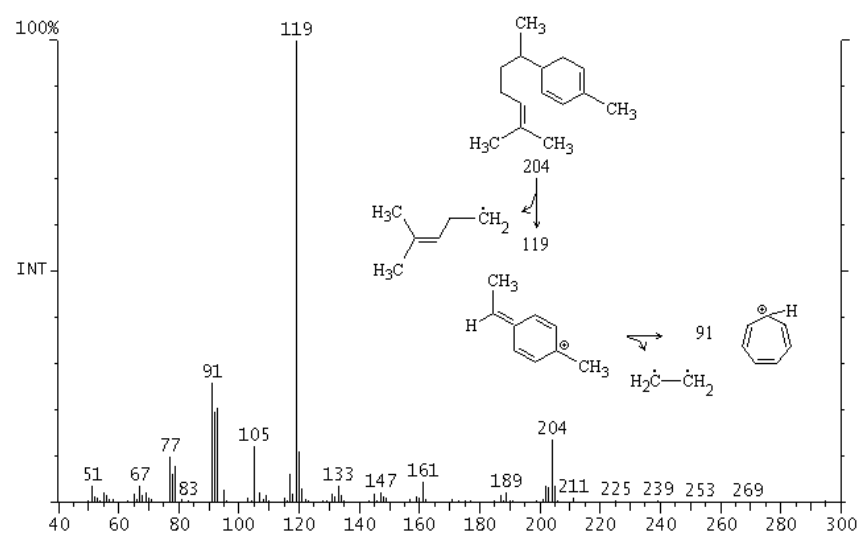

Condições cromatográficas: coluna DB-5 $(30 \mathrm{~m} \times 0,25 \mathrm{~mm}$; $0,25 \mathrm{~mm})$; gás de arraste hélio; temperatura do injetor $220^{\circ} \mathrm{C}$, do detector $250^{\circ} \mathrm{C}$ e da coluna $40^{\circ} \mathrm{C}$ por $5 \mathrm{~min}$, aumento de $8^{\circ} \mathrm{C} / \mathrm{min}$ até $130^{\circ} \mathrm{C}$, aumento de $30^{\circ} \mathrm{C} / \mathrm{min}$ até $200^{\circ} \mathrm{C}, 200^{\circ} \mathrm{C}$ por $5 \mathrm{~min}$. Condições de espectrometria de massa: detector de ion trap, temperatura da fonte
$170^{\circ} \mathrm{C}$, temperatura da interface $275^{\circ} \mathrm{C}$, ionização $70 \mathrm{eV}$ e corrente de emissão $250 \mathrm{~mA}$

FIGURA 6. Espectro de massa e possiveis fragmentos do zingibereno obtido na espectrometria de massa.

\section{4 - CONCLUSÕES}

Na otimização das metodologias de extração por fase sólida e cromatografia gasosa, as soluções dos padrões de borneol, limoneno e linalol na faixa de 30 a $1000 \mu \mathrm{g} / \mathrm{L}$ exibiram uma resposta linear da área dos picos separados por cromatografia gasosa, em função da concentração. A condição cromatográfica que melhor separou os picos dos padrões de borneol, limoneno e linalol, além dos nove picos predominantes das amostras de cúrcuma foi com a programação de temperatura da coluna de $80^{\circ} \mathrm{C}$ por $2 \mathrm{~min}$, aumento de $8^{\circ} \mathrm{C} / \mathrm{min}$ até $130^{\circ} \mathrm{C}, 130^{\circ} \mathrm{C}$ por $1 \mathrm{~min}$, aumento de $30^{\circ} \mathrm{C} / \mathrm{min}$ até $200^{\circ} \mathrm{C}$ e manutenção a $200^{\circ} \mathrm{C}$ por $5 \mathrm{~min}$. O tempo de $5 \mathrm{~min}$ foi o ideal para a partição dos compostos voláteis, a partir dos estudos realizados com os padrões. O emprego de $0,1 \mathrm{~g}$ da amostra e a temperatura de $70^{\circ} \mathrm{C}$ foi a melhor dentre as condições estudadas para a partição dos nove compostos voláteis predominantes na amostra. A temperatura de $220^{\circ} \mathrm{C}$ e o tempo de $5 \mathrm{~min}$ foram as melhores condições para a dessorção dos compostos voláteis da cúrcuma. 
A análise dos espectros de massas obtidos indicou a presença de sabineno, 1,8-cineol, 1,4-terpineol, arcurcúmeno, zingibereno, $\beta$-sesquifelandreno e ar-turmerona. Dentre estes, ar-curcúmeno, zingibereno, $\beta$ sesquifelandreno e ar-turmerona corresponderam, respectivamente, aos picos $3,4,6$ e 7 das amostras de cúrcuma analisadas.

\section{5 - REFERÊNCIAS BIBLIOGRÁFICAS}

[1] AOAC - ASSOCIATION OF OFFICIAL ANALYTICAL CHEMISTS. Official methods of analysis of AOAC international. 16 ed. Arlington: AOAC International, 1995. v. 2, p. 43.1-43.13.

[2] ARTHUR, C.; KILlaM, L.M.; BUCHHOLZ, K.D.; PAWLISZYN, J. Automation and optimization of solidphase microextraction. Analytical Chemistry, v. 64, p. 1960-1966, 1992.

[3] ARTHUR, C.; PAWLISZYN, J. Solid phase microextraction with thermal desorption using fused silica optical fibers. Analytical Chemistry, v. 62, p. 2145-2148, 1990.

[4] BAMBIRRA, M.L.A.; JUNQUEIRA, R.; GLÓRIA, M. B. A. Influence of Post Harvest Processing Conditions on Yield and Quality of Ground Turmeric (Curcuma longa L.). Brazilian Archives of Biology and Technology, v. 45. p. 423-429, 2002.

[5] CHATTERJEE, S.; VARIYAR, P.S.; GHOLAP, A.S.; PADWAL-DESAI, S.R.; BONGIRWAR, D.R. Effect of gamma-irradiation on the volatile oil constituents of turmeric (Curcuma longa). Food Research International, v. 33. p. 103-106, 2000.

[6] CHOUdhuRY, S.N.; GHOSH, A. C.; SAIKIA, M.; CHOUDHURY, M.; LECLERCQ, P.A. Volatile constituents of the aerial and underground parts of Curcuma aromatica Salisb. from India. Journal Essential Oil Research, v. 8, p. 633-638, 1996.

[7] GOVINDARAJAN, V.S. Turmeric - chemistry, technology and quality. CRC Critical Reviews in Food Science Nutrition, v. 12, p. 199-301, 1980.

[8] KELKAR, N. C.; RAO, B.S. Indian essential oils V. Essential oil from the rhizomes of Curcuma longa L. J. Indian Inst. Sci., 17 A: 17, 1934 apud GOVINDARAJAN, V. S. Turmeric - chemistry, technology and quality. CRC Critical Reviews in Food Science Nutrition, v. 12, p. 199-301, 1980.

[9] MAGDA R.R. Turmeric: a seasoning, dye, and medicine. Food Marketing and Technology, v. 8, p. 9-10, 1994.

[10] MARTINS, M.C.; RUSIG, O. Cúrcuma - um corante natural. Boletim SBCTA, v. 26, p. 53-65, 1992.

[11] MAUL, A.A.; POLAKIEWICZ, B.; OLIVEIRA, M.N. Extraction of turmeric rhizomes (Curcuma domestica Valeton, Zingiberaceae) colorings using supercritical carbon dioxide. In: IUPAC INTERNATIONAL
CONFERENCE ON BIODIVERSITY, 2, 1999. Livro de resumo. Belo Horizonte: IUPAC, 1999. p.125.

[12] MÉNDEZ, A.L.C.; CORRÊA, N.C.F.; MACHADO, N.T.; ARAÚJO, M.E.; MEIRELES, M.A.A. Extraction of curcumins from the rhizomes of turmeric (Curcuma longa L) with supercritical $\mathrm{CO}_{2}$. In: IUPAC INTERNATIONAL CONFERENCE ON BIODIVERSITY, 2, 1999. Livro de resumo. Belo Horizonte: IUPAC, 1999. p.140.

[13] OGUNTIMEIN, B.O.; WEYERSTAHL, P.; MARSCHALLWEYERSTAHL, H. Essential oil of Curcuma longa L. leaves. Flavour and Fragrance Journal, v. 5, p. 89$90,1990$.

[14] OLIVEIRA, V.P.; GHIRALDINI, J.E.; SACRAMENTO, C.K. O cultivo de plantas produtoras de corantes. Revista Brasileira de Corantes Naturais, v 1, p. 232237, 1992

[15] PIMENTEL-GOMES, F. Curso de estatística experimental. 14. ed. Piracicaba: F. Pimentel-Gomes Ed., 2000. 477 p.

[16] RICHARD, H.; LOO, A. Composition des extraits d'épices et herbes aromatiques. In: RICHARD, H. (Coord.). Épices et aromates. Paris: TEC \& DOC - Lavoisier, 1992. p.213-238.

[17] RICHMOND, R.; POMBO-VILLAR, E. Gas chromatographymass spectrometry coupled with pseudo-sadtler retention indices, for the identification of components in the essential oil of Curcuma longa L. Journal of Chromatography A, v. 760, p. 303-308, 1997.

[18] SANTOS, F.J.; GALCERAN, M.T.; FRAISSE, D. Application of solid-phase microextraction to the analysis of volatile organic compounds in water. Journal of Chromatography A, v. 742, p. 181-189, 1996.

[19] SHARMA, R.K.; MISRA, B.P.; SARMA, T.C.; BORDOLOI, A.K.; PATHAK, M.G.; LECLERCQ, P.A. Essential oils of Curcuma longa L. from Bhutan. Journal Essential Oil Research, v. 9, p. 589-592, 1997.

[20] SONG, J.; GARDNER, B. D.; HOLLAND, J.F.; BEAUDRY, R.M. Rapid analysis of volatile flavor compounds in apple fruit using SPME and GC/time of flight mass spectrometry. Journal Agriculture Food Chemistry, v. 45, p. 1801-1807, 1997.

[21] SOUZA, C. R. A.; GLÓRIA, M. B. A. Chemical analysis of turmeric from Minas Gerais, Brazil and comparison of methods for flavor free oleoresin. Brazilian Archives of Biology and Technology, v. 41, p. $218-224,1998$.

[22] VARIYAR, P.S.; GHOLAP, A.S.; THOMAS, P. Effect of g-irradiation on the volatile oil constituints of fresh ginger (Zingiber officinale) rhizome. Food Research International, v. 30, p. 41-43, 1997.

[23] YANG, X.; PEPPARD, T. Solid-phase microextraction for flavor analysis. Journal of Agriculture Food Chemistry, v. 42, p. 1925-1930, 1994.

[24] ZWAVING, J.H.; BOS, R. Analysis of the essential oils of five Curcuma species. Flavour and Fragrance Journal, v. 7, p. 19-22, 1992. 